\title{
Effect of Physician Profiles and Academic Detailing on Cost and Utilization of Selective Serotonin Reuptake Inhibitors
}

OBJECTIVE: To evaluate the effect of physician profiles and academic detailing on cost and utilization of selective serotonin reuptake inhibitors (SSRIs) in a managed care population.

DESIGN: A retrospective review of 153,072 SSRI pharmacy prescription claims from January 1997 through December 2000.

SETTING: The health plan is a staff-model managed care organization (MCO) in the central Texas region with more than 150,000 members and over 500 participating physicians.

INTERVENTIONS: (1) Physician prescribing (profile) reports were implemented in November 1998 and thereafter distributed quarterly to MCO physicians. (2) These reports were supplemented with academic detailing at a majority of the health plan clinics. (3) SSRI utilization data were added to the prescriber profile reports in the first quarter of 1999.

MAIN OUTCOME MEASURES: Prescriptions (Rxs), patients (utilizing members), days of therapy, drug (ingredient) cost and member-months were measured. The principal outcome measures are SSRI share percentages as assessed by days of therapy, number of patients and number of prescriptions. Utilization and drug cost per-member-per-month (PMPM) and perpatient-per-year (PPPY) are reported for 2 years before and 2 years after physicians began receiving the prescribing reports.
RESULTS: In 1997, the Rx market share for non-fluoxetine ("other") SSRIs at the health plan was below the national average ( $53.8 \%$ versus $55.4 \%$, respectively). In the year immediate post-intervention (CY 1999), the health plan market share for the other SSRIs exceeded the national average ( $68.0 \%$ versus $64.5 \%$, respectively) and continued to grow in CY 2000 to $77.0 \%$. PMPM utilization for all SSRls increased from 0.535 days PMPM in 1997 to 0.763 days PMPM in CY 2000, but the average cost of SSRI drug therapy declined from $\$ 2.43$ per day for CY 1998 to \$2.25 per day of therapy in CY 1999 and to $\$ 2.16$ per day of SSRI therapy in CY 2000 , a relative difference of $7.4 \%$ in savings per day of SSRI therapy in CY 1999 and an additional $4.0 \%$ in CY 2000. Net health plan savings compared to national trend in the relative $\mathrm{Rx}$ share of fluoxetine among SSRls was $\$ 206,644$ over the 2-year period CY 1999 through CY 2000 or $\$ 0.61$ per health plan member per year (PMPY)

CONCLUSIONS: The combination of physician prescribing profiles and academic detailing was associated with a decrease in the utilization of fluoxetine, a more expensive SSRI, and an increase in utilization of less expensive (other) SSRIs. The average cost per day of therapy for SSRls was reduced without altering the formulary or the copay status of the SSRIs. Net health plan savings were \$57,191 (\$0.34 PMPY) in CY 1999 and \$149,453 (\$0.89 PMPY) in CY 2000.

KEYWORDS: Physician profiles, academic detailing, selective serotonin reuptake inhibitors

J Managed Care Pharm 2002: 23-31
KRISTA K. YOKOYAMA, PHARM.D., is Pharmacoeconomic and Outcomes Research Fellow at Scott \& White Memorial Hospital/University of Texas at Austin/Novartis Pharmaceuticals Corporation, East Hanover, New Jersey. QUYNHCHAU D. DOAN, R.PH., M.S., was Pharmacoeconomic and Outcomes Research Fellow at Scott \& White Memorial Hospital, and now is Doctoral Candidate, Division of Pharmacy Administration, College of Pharmacy, University of Texas, Austin, Texas. HOA T. PHAM, PHARM.D., was Pharmacoeconomic and Outcomes Research Fellow at Scott \& White Memorial Hospital, and now is Manager, Global Health Outcomes at Pharmacia Corporation, Peapack, New Jersey. PAUL J. GODLEY, PHARM.D., BCPS, FASHP, is Assistant Director of Patient Care Services, JOHM M. JACKIEMIEC, R.PH., is Clinical Specialist, BILLY W. WOODWARD, R.PH., is Director of Pharmacy, all at Scott \& White Memorial Hospital, Temple, Texas. CRAIG CLANTON, M.D., is Associate Medical Director for Medical Operations, Networks and Pharmacy, Scott $\&$ White Health Plan, and Assistant Professor of Family \& Community Medicine, TAMU Health Science Center College of Medicine, Temple, Texas.

Address correspondence to Paul J. Godley, Pharm.D., Assistant Director of Patient Care Services, Department of Pharmacy, Scott \& White Memorial Hospital, Department of Pharmacy, 2401 South 31st St., Temple, TX 76508,E-mail:pgodley@swmail.sw.org.

The assistance of James P. Wilson, Pharm.D., Ph.D., FASHP, Associate Professor and Chair, Pharmacy Practice, University of Texas, Austin College of Pharmacy, also is acknowledged.

Copyright $\odot 2002$ Academy of Managed Care Pharmacy, Inc. All rights reserved.

\begin{tabular}{c}
\hline by Krista K. Yokoyama, \\
QuynhChau D. Doan, \\
Paul J. Godley, Hoa T. Pham, \\
John M. Jackimiec, Billy W. Woodward \\
and Craig Clanton
\end{tabular}

$\mathrm{P}$ hysician profiling is a feedback mechanism that has been used by many institutions, particularly hospitals and managed care organizations, to educate and possibly influence physicians' practice patterns. Physician profiling and "report cards" are likely to increase in frequency of use due to increased emphasis on managed care organization (MCO) performance measures, including National Committee for Quality Assurance (NCQA) performance measures to accredit health plans and Health Plan Employer Data and Information Set (HEDIS) measures.

Physician profile reports used in many organizations contain information as diverse as utilization of clinical procedures, laboratory tests, prescriptions drugs, office visits, hospital admissions, and specialty referrals. Printed materials aimed at educating physicians regarding various prescribing or practice topics commonly accompany these reports. Physician prescription profiles are used to inform physicians of their prescribing patterns for targeted drug classes or disease states, encourage formulary prescribing, enhance compliance with disease treatment algorithms, and promote cost and care management.

The use of physician profiles has had mixed results in affecting prescribing or clinical practice patterns. In a meta-analysis of 12 randomized, controlled clinical trials, Balas et al. reported that the peer-compari-

Editor's note: The study period for this research ended in December 2000, nearly 8 months before the market availability of generic fluoxetine in August 2001 
son feedback mechanism yielded only a modest effect on practice patterns, and the potential cost-savings from changes in physician behavior did not always outweigh the cost of providing the feedback. ${ }^{1}$ Limited research has been published on the sole impact of physician profiles or the combined effect of physician profiles and academic detailing on prescribing habits and practice patterns.

In studies from the 1980s, the use of physician profiles resulted in decreased prescription expenditures, more appropriate medication utilization, and decreased utilization of laboratory testing.${ }^{4}$ In contrast, other studies demonstrated that physician profile reports did not have significant effects on prescribing or practice patterns..$^{5-9}$ These studies combined the profile reports with other forms of intervention, such as written educational information ${ }^{5,6}$ or patient-specific prescribing recommendations. ${ }^{7.8}$ The results from these studies do not appear to support the hypothesis that multiple interventions would create linear, if not additive, effects on utilization and cost outcome measures. Hershey et al. and Wones demonstrated that knowledge of the cost of procedures alone does not necessarily influence physician behavior. ${ }^{6,9}$

A 1993 survey conducted by the American Medical Association showed that $53 \%$ of the physicians received some type of profile reports. ${ }^{10}$ Physicians who were either employed by or contracted with health maintenance organizations (HMOs) were more likely to receive feedback regarding their practice patterns, patient outcomes, and satisfaction with care. The effectiveness of physician profiling may be influenced by the practice setting of physicians. Staff-model MCOs may be particularly conducive to such feedback mechanisms because of financial risk-sharing with physicians. Brufsky et al. reported feedback interventions were effective in changing prescribing behavior for both staff- and group-model physicians; however, only modest changes were observed for the group-model physicians. ${ }^{7}$ In a study by Schectman et al., interventions resulted in favorable changes in prescribing patterns of group-model HMO physicians, whereas the interventions were ineffective for network-model physicians. ${ }^{5}$

The research in this Texas health plan was undertaken to assess the impact on cost and utilization of selective serotonin reuptake inhibitors (SSRIs) associated with the distribution of physician profiles in combination with periodic counter-detailing education conducted in group settings with participating physicians. Nationally, antidepressants were the top-selling drug class by HMO dollar volume and prescriptions per 1,000 members for 1998, 1999, and 2000.11-13 Accordingly, the ability to reduce the average cost per day of drug therapy for depression was of interest in this $\mathrm{MCO}$ and in the managed care industry in general.

\section{Methods}

\section{Objective}

A retrospective analysis of pharmacy claims was used to evaluate the effect of physician profiles and academic detailing on prescribing trends of primary care providers (family practice and internal medicine) for SSRIs, as measured by drug cost and utilization (prescriptions and days of therapy) per-member-permonth (PMPM) and per-patient-per-year (PPPY).

\section{Setting}

The study was conducted using data from a staff-model MCO located in central Texas that served approximately 135,000 members in 1997 and grew to approximately 168,500 members in 2000. Over 500 physicians were employed by this MCO including 158 family practice and internal medicine physicians at 19 regional clinics in a coverage area of about 20,000 square miles.

\section{Physician Profile}

Two types of physician reports were provided to MCO physicians during the intervention period beginning at year-end 1998. One physician report provided physician-specific prescribing information. This report included average cost per prescription, average number of prescriptions per patient, and average total prescription costs per patient. It was produced for each physician as an individual and for the physicians as a group at each regional clinic (Figure 1, Physician Report A). The data used for these profiles held physicians accountable for prescriptions written and refills authorized by physician's assistants, nurse practitioners, etc., regardless of practice site. Data for physicians who practiced at more than one regional clinic were analyzed according to their primary workplace. Physician-specific top 20 lists of drugs by cost and utilization and a top 20 list of non-formulary medications were also provided.

The second type of physician report, Physician Report B (Figure 2), examined the prescribing trends of the regional clinic by calendar quarter. Specific drug classes were targeted for benchmarking to other clinics, comparing utilization patterns per clinic and per physician. These drug classes were the most costly drug classes by volume or by unit cost and included SSRIs, antihypertensives, and gastrointestinal medications.

\section{Implementation of Physician Profiles}

Physician profiles were initially implemented in November 1998 and were updated and distributed quarterly. From Physician Report A, physicians could see how their utilization of SSRI drugs ranked among the top 20 drugs by cost and by number of prescriptions. Prior to implementation of the physician profiles, SSRIs were the second highest selling drug class by dollar volume and the third highest by prescription volume in the health plan. To further emphasize this information, SSRI trend data were provided in Physician Report B. SSRI trend data were added to the physician profiles during the first quarter of 1999.

Academic detailing was provided to the largest 13 of the 19 regional clinics (150 family practice and internal medicine physicians). The quarterly academic detailing program had been implemented for several years prior to physician profiling. Although not mandatory, attendance at these meetings was 
Effect of Physician Profiles and Academic Detailing on Cost and Utilization of Selective Serotonin Reuptake Inhibitors

FIGURE 1 Physician Report A - Department Summary 302000
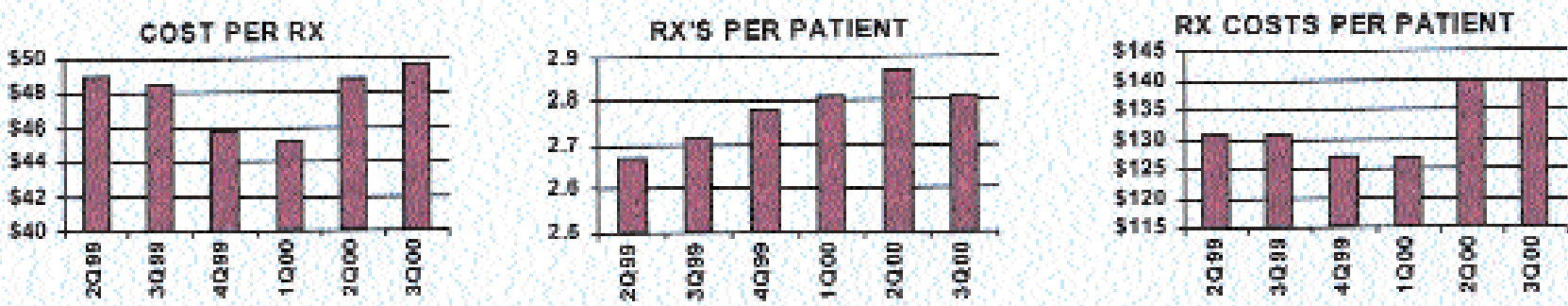

\begin{tabular}{|c|c|c|c|c|c|c|c|c|c|c|}
\hline \multicolumn{4}{|c|}{ TOP 20 DRUGS BYCOST } & \multicolumn{3}{|c|}{ TOP NON.FORMULARY } & \multicolumn{4}{|c|}{ TOP 20 DRUGS BYRX'S } \\
\hline & & AVE & TOTAL & & AVE & TOTAL & & & AVE & TOTAL \\
\hline DRUG & $R X ' S$ & QTY & $\cos T$ & DRUG & QTY & $\cos T$ & DRUG & $R X ' S$ & QTY & $\cos T$ \\
\hline ZOCOR & & & & ORTHO TRLCYCLEN & & & PRINVIL & & & \\
\hline PREVACD & & & & LIRTOR & & & ALLEGRA & & & \\
\hline PFUNNLL & & & & ORTHO NOVUM & & & ZOLOFT & & & \\
\hline ALLEGRA & & & & ORTHO-CYCLEN & & & FLONASE & & & \\
\hline ZOLOFT & & & & CLAFIN & & & ZOCOR & & & \\
\hline FLONASE & & & & DEIROL & & & LEVOTHROID & & & \\
\hline GLUCORTAGE & & & & QUNDAL HD & & & ATENOLOL & & & \\
\hline NOFVASC & & & & BERACW & & & NAPROXEN & & & \\
\hline ACIHHEX & & & & PERPHENAMT & & & PFENARN & & & \\
\hline PROZAC & & & & CONERA-HS & & & RANITDNE & & & \\
\hline
\end{tabular}
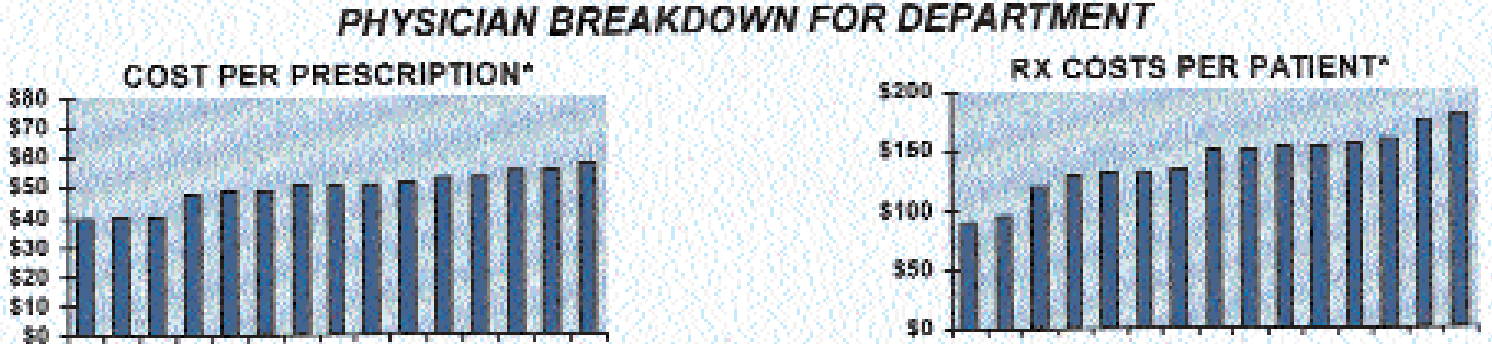

"Each bar represents Individual PCP's rank among all PCPs in this speclfic clinic

FIGURE 2 Physician Report B - 1999-2000 Department Trend

SSRI Drugs per Clinic

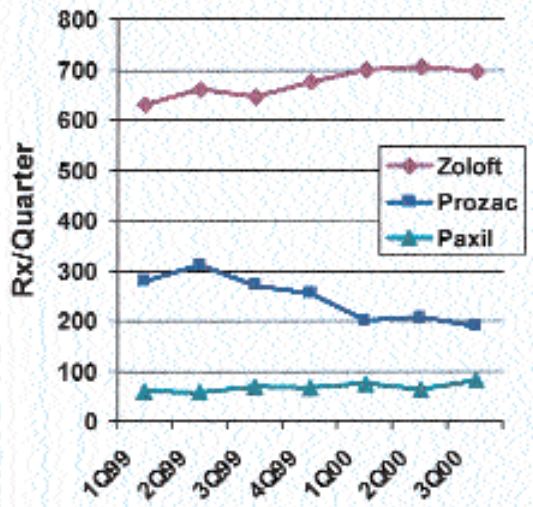

Quarter
SSRI Drugs per Physician

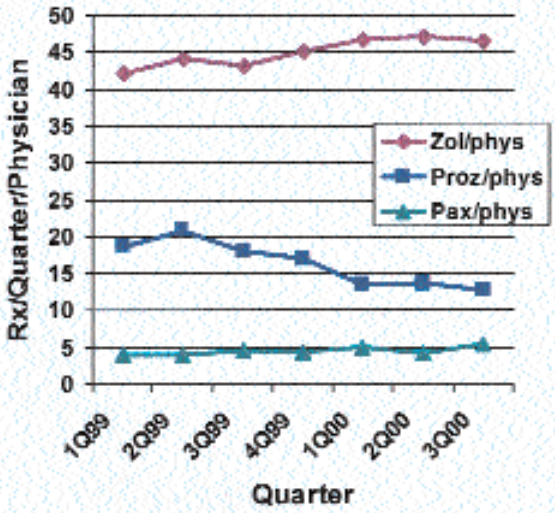


highly recommended. Pharmacists incorporated discussions of the physician profiles and trend data for SSRI utilization with formulary and pricing concerns, continuous quality improvement initiatives, therapeutic interchange programs, etc. Clinic department heads were responsible for further emphasizing these issues with their staff physicians.

All health plan physicians, regardless of specialty or clinic, received Physician Report A through the mail. The mailed Report A contained physician-specific utilization data. During the quarterly clinic meetings, physicians were provided with Report B as well as a revised copy of Physician Report A, with utilization data aggregated for the clinic.

\section{Pharmacy Claims Analysis}

SSRI pharmacy claims data from January 1997 through December 2000 were obtained. Liquid formulations for fluoxetine and paroxetine were not included in the analysis. Additionally, a new formulation of fluoxetine (Sarafem), utilized in premenstrual dysphoric disorder (PMDD) and first dispensed to health plan patients in the third quarter of 2000, was not included in the claims analysis. Sarafem was not on the health plan formulary. Additionally, at the time of this study, neither the once weekly formulation of fluoxetine (Prozac Weekly) nor generic fluoxetine was available.

All SSRIs were on the formulary at the same copay level during the study period except fluvoxamine, which required prior authorization and a higher copay. Fluoxetine had a higher cost to the MCO when compared to the other SSRIs. None of the SSRIs were considered a "preferred" agent. SSRIs included citalopram, fluoxetine, fluvoxamine, paroxetine and sertraline. Citalopram was first dispensed to health plan patients in the fourth quarter of 1998.

Analyses of PPPY prescription utilization and PPPY drug costs were based on SSRI utilizing members (patients) and actual (adjudicated) prescription drug (ingredient price) without the dispensing fee and before member copayment. Data were initially calculated for each calendar quarter. Annual utilization and drug cost were determined by aggregating the quarterly data. The drug cost data do not reflect manufacturer rebates or pharmacy contractual discounts. Prescriptions dispensed by health plan pharmacies and community network pharmacies were adjudicated in the same manner, employing the same drug cost formula. All drug costs were adjusted to 1998 dollars as estimated from the Consumer Price Index (CPI) rate of inflation. ${ }^{14}$ SSRI drug prices changed at a rate similar to the CPI during the time period of this study. Specifically, actual 1997 cost data were multiplied by $1.55 \%$, actual 1999 cost data were divided by $2.21 \%$, and actual 2000 cost data were divided by 5.64\%.

Health plan market shares for the SSRIs were compared with national data obtained from the Express Scripts 1999 Drug Trend Report and 2000 Drug Trend Report. ${ }^{15,16}$ Data in these reports represented prescription utilization of between 8.8 and 9.6 million managed and non-managed care commercial members nationwide from 1995 to 2000. Annual cost savings to the health plan were determined from the reduction in average SSRI cost per day, in 1998 dollars, multiplied by the total number of SSRI days of therapy for CY 1999 and CY 2000.

\section{Results}

\section{MCO Pharmacy Claims}

A retrospective review of prescription claims yielded 153,072 SSRI prescriptions dispensed between January 1, 1997, and December 31, 2000, net of claim reversals. Table 1 shows the number of SSRI prescriptions for this health plan, including prescription volume, days supply, average daily dose, average drug cost, number of SSRI utilizing members (patients), member-months and the measures derived from these data.

\section{SSRI Market Share}

Three measures were employed to determine SSRI market share - prescriptions, days of therapy and number of patients. A comparison of national and health plan prescription ( $\mathrm{Rx})$ market share changes for fluoxetine can be seen in Figure 3. MCO market share for fluoxetine as measured by number of Rxs was above the national average in 1997 (46.2\% versus 44.6\%, respectively). In 1999, the Rx market share for fluoxetine was below the national average (32.0\% versus $35.5 \%$, respectively), and continued to decrease in 2000 (23.0\% versus $30.6 \%$, respectively), a relative market share reduction of $50 \%$ from 1997 to 2000. The effect of the mail-order option for this health plan appears minimal since the SSRI market share as measured by days of therapy is nearly equal to the market share as measured by Rxs (Table 1 and Figure 4). The health plan market share Rx change for each SSRI is shown in Figure 5. Market share as measured by the percentage of patients (Figures 4 and 6 and Table 1) shows a relative share reduction (erosion) of $52 \%$ for fluoxetine from 1997 to 2000 , from $41.5 \%$ of patients in 1997 to $20.1 \%$ of patients in 2000.

\section{SSRI Utilization}

The primary measures of SSRI utilization were prescriptions, days of therapy and patients, with derivative measures calculated from prescriptions and days of therapy per SSRI patient and per-member-month (PMPM). SSRI utilization increased in the health plan during this four-year period. The SSRI days of therapy PMPM increased by 43\% from 0.535 days PMPM in 1997 to 0.763 days PMPM in 2000 (Figure 7), and the percentage of patients in the health plan using SSRIs increased from $4.8 \%$ of members in 1997 to $6.4 \%$ of members in 2000 (Table 1), a relative increase of 33\%. Utilization of SSRIs per patient remained relatively stable for the SSRI class during the study period. As shown in Figure 8, there was only a modest increase $(6 \%)$ in the average days of therapy per SSRI patient-per-year (PPPY), rising from 134 days of therapy in 1997 to 142 average days of ther- 
TABLE 1 Health Plan Cost and Utilization Data for SSRIS

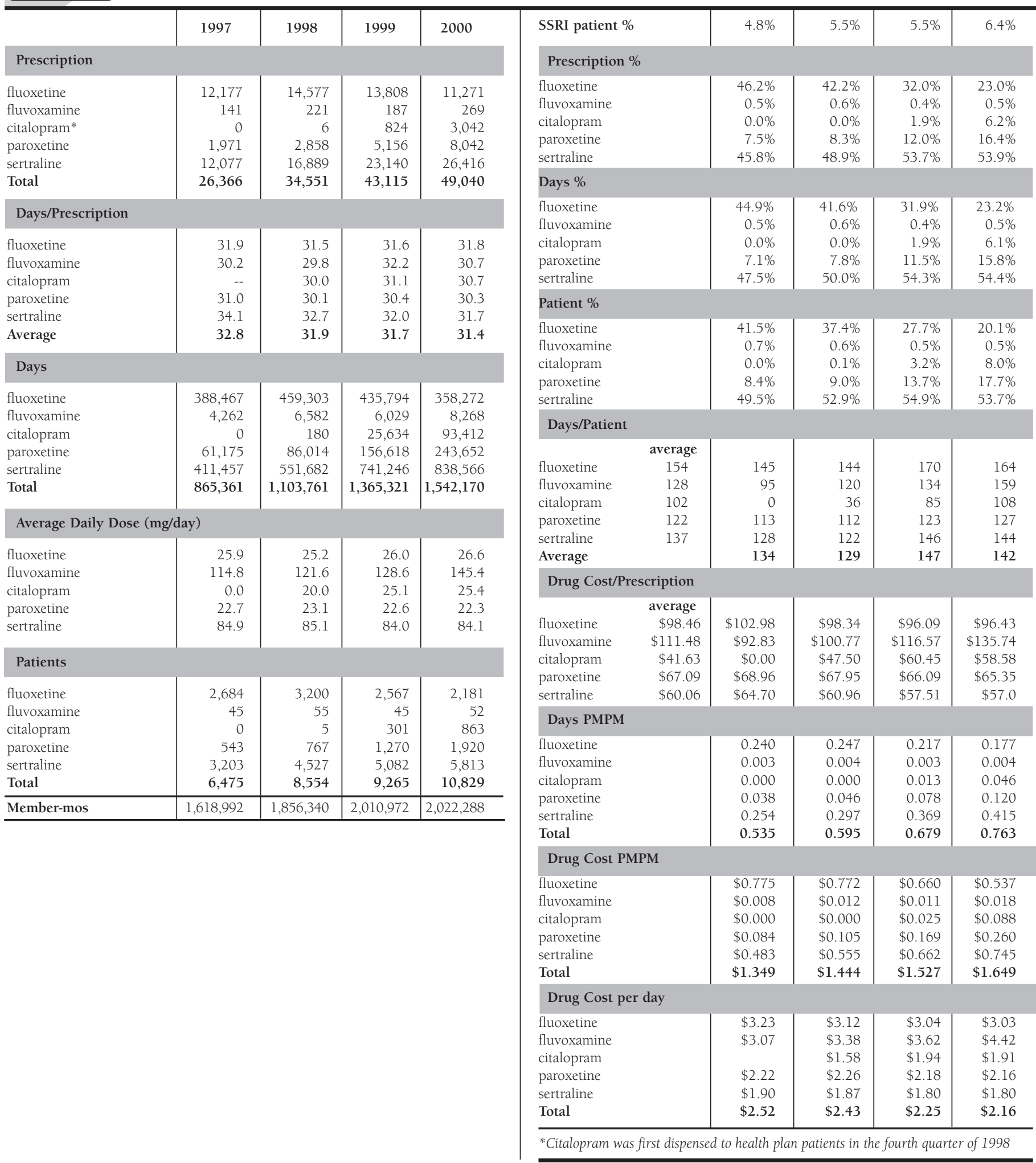


FIGURE 3 Prescription Market Share of Fluoxetine ${ }^{15,16}$

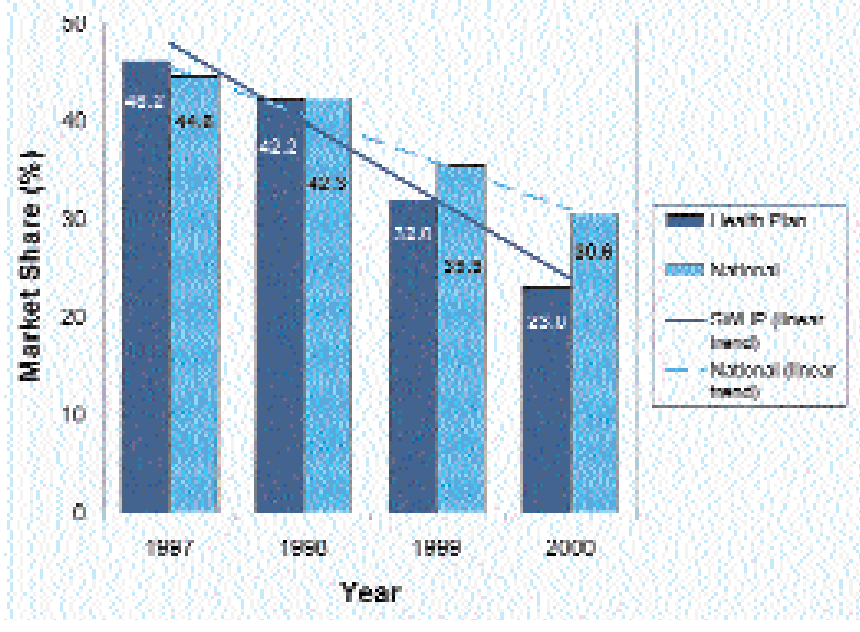

FIGURE 5 Prescription Market Share of SSRIS by Calendar Quarter

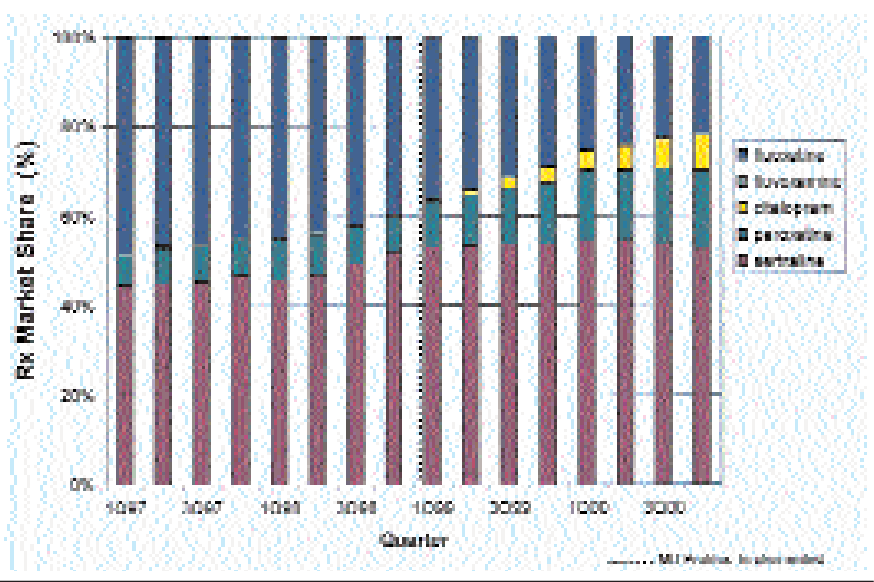

FIGURE 7 SSRI Days of Therapy PMPM

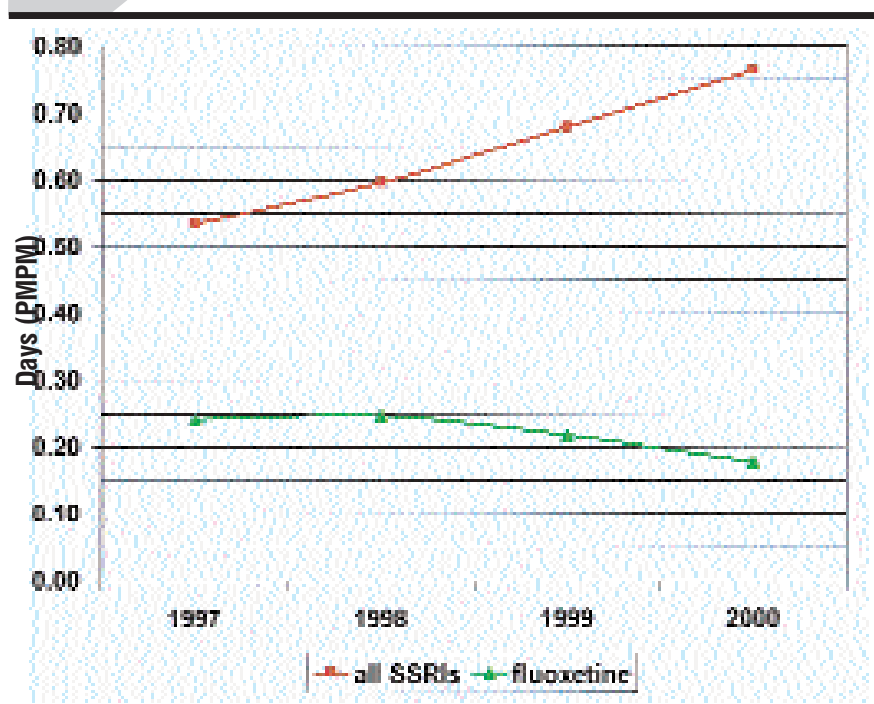

FIGURE 4 Fluoxetine Market Share in Days vs. Patients vs. Prescriptions

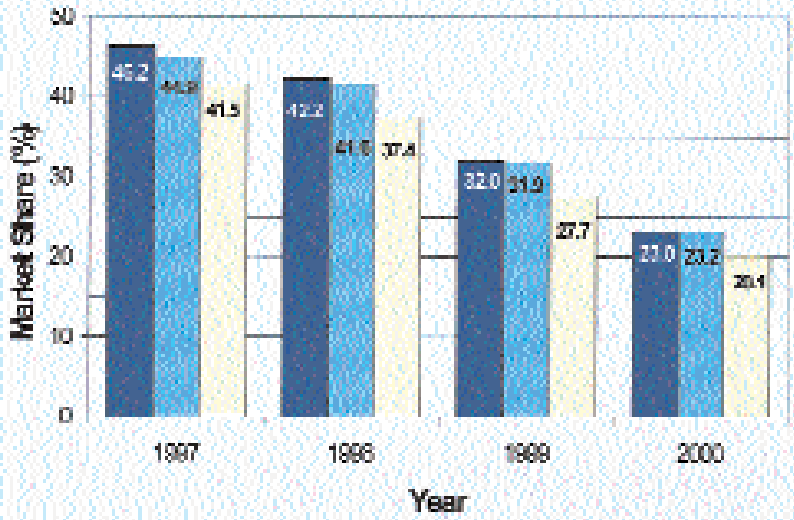

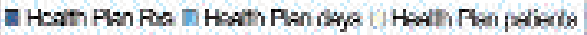

\section{FIGURE 6 Patient Market Share of SSRIs}

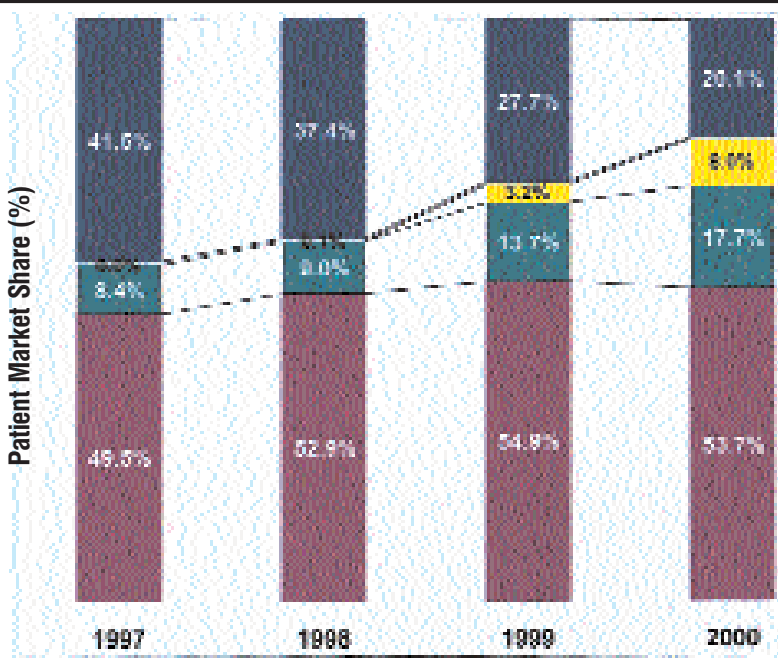

nestraline Eparoxntine citalopram fluvoxamine Efluoxetine

FIGURE 8 SSRI Utilization (Days PPPY) ${ }^{\mathrm{a}, \mathrm{b}}$

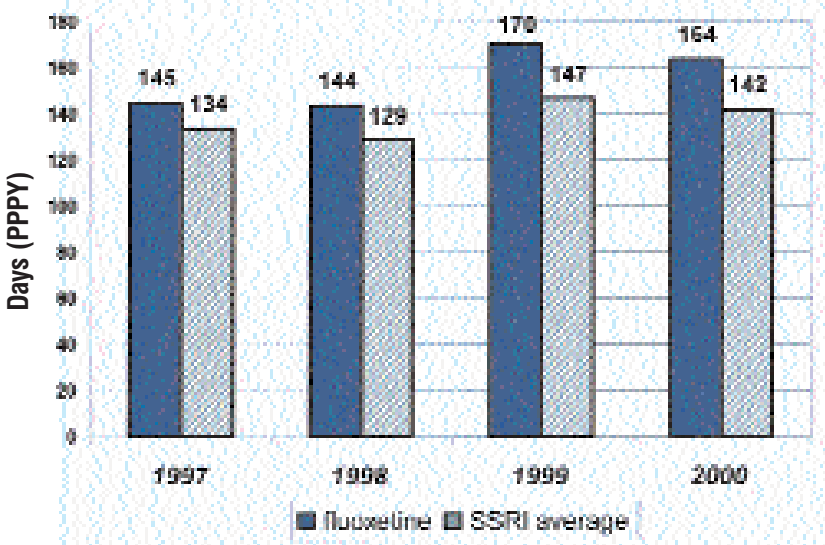

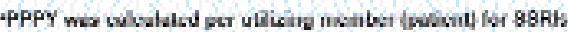

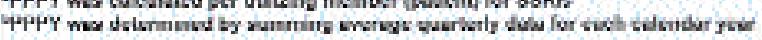


apy in 2000. Days of therapy per fluoxetine patient increased 13\% from 145 days in 1997 to 164 days in 2000. Fluoxetine days PMPM declined by 26\% from 0.240 days PMPM in 1997 to 0.177 days PMPM in 2000.

\section{SSRI Drug Cost}

Drug cost for SSRI prescriptions in this health plan increased from \$2.68 million in CY 1998 to \$3.33 million in CY 2000. Despite this increase, expenditures for fluoxetine decreased from $\$ 1.43$ million in 1998 to $\$ 1.09$ million in CY 2000. The average cost per day of SSRI drug therapy declined by $11.1 \%$ (in 1998constant dollars) from an average drug cost per day of $\$ 2.43$ in CY 1998 to $\$ 2.16$ per day of SSRI therapy in CY 2000 (Figure 9). The average cost of SSRI drug therapy declined by $7.4 \%$ in $\mathrm{CY}$ 1999 and by 4.0\% from CY 1999 to CY 2000.

Despite the decrease in average drug cost per day of SSRI therapy during the study period, health plan total cost for SSRI drug therapy increased due to an increase in utilization, as measured by the number of patients and days of therapy PMPM. SSRI drug cost PMPM rose 22\% from \$1.35 PMPM in CY 1997 to \$1.65 PMPM in CY 2000 (Figure 10), while fluoxetine cost PMPM declined by $31 \%$, from $\$ 0.78$ PMPM in CY 1997 to $\$ 0.54$ PMPM in CY 2000, proportionate to the (26\%) decline in fluoxetine utilization PMPM (Table 1).

\section{Discussion}

SSRIs have consistently been among the top 10 drug classes in expenditures and prescription volume for MCOs. Nationally, utilization of fluoxetine has decreased as less expensive SSRIs entered the market. This health plan experienced a similar trend in the declining ratio of fluoxetine to total SSRI utilization from 1997 to 2000 which was magnified by the intervention program. This program combined physician profiles with "report cards" on SSRI utilization and academic counter-detailing that included information about the relative costs of SSRI drug therapy. This health plan experienced a 50\% decline in relative fluoxetine share of SSRI prescriptions, from $46.2 \%$ fluoxetine Rxs in CY 1997 to $23.0 \%$ in CY 2000, compared to a national market share erosion of $31 \%$, from $44.6 \%$ fluoxetine Rxs in CY 1997 to 30.6\% in CY 2000. This reduction in the relative ratio of fluoxetine to total SSRI utilization helped push down the average daily cost of SSRI therapy by $7.4 \%$, from $\$ 2.43$ in CY 1998 to $\$ 2.25$ in CY 1999 and by an additional 4.0\% in CY 2000 to $\$ 2.16$, saving the health plan $\$ 245,760$ (in 1998 dollars) in CY 1999 and $\$ 415,385$ in CY 2000, or a combined twoyear cost savings of $\$ 661,145$.

Based upon comparison to national market share erosion for fluoxetine, the intervention program in this health plan employing physician profiles and counter-detailing was associated with $37 \%$ of the change, a relative change of $50 \%$ decline in fluoxetine Rx market share from 1997 to 2000 versus 31\% decline in the national average fluoxetine Rx market share. Therefore, the com-
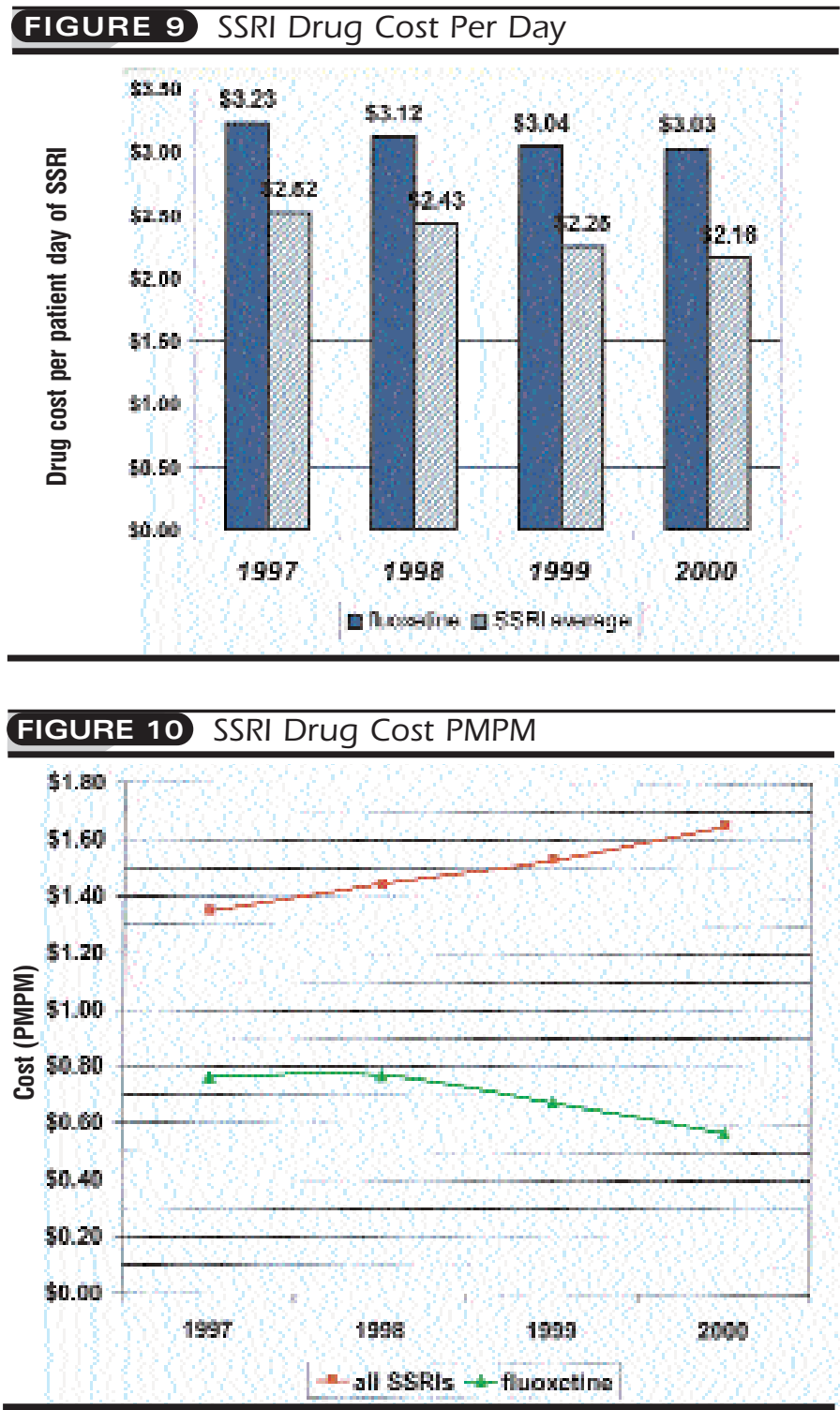

bined intervention program at this health plan saved approximately $\$ 57,191$ (\$0.34 PMPY) in CY 1999 and \$149,453 (\$0.89 PMPY) in CY 2000, or two-year savings of about $\$ 206,644$ or an average of $\$ 0.61$ per health plan member per year (PMPY).

While removing fluoxetine from the MCO formulary would have effectively decreased its utilization, the MCO Pharmacy and Therapeutics Committee felt that a forced switch from fluoxetine to other SSRIs was not desirable for this class of medications. This decision was influenced by concern over interrupting antidepressant drug therapy in patients stabilized on fluoxetine. The support of the health plan administrators and clinical thought leaders was also important to invoke a favorable change in prescribing habits as well as successfully implementing physician profiles. The results in this staff-model MCO 
may not be representative of the potential effects of this intervention in other MCO models such as independent-practice association MCOs. Furthermore, the senior managers and thought leaders at this health plan provided feedback during the development of the reports. The content of these reports was continuously updated in response to physician inquiries and changes in formulary and physician status. This process for implementing physician profiles appeared to favorably affect physician confidence in the validity and reliability of the information contained in the reports.

The positive results seen in this study are generally consistent with the work of others who have demonstrated that education efforts utilizing data (report cards) and counter-detailing (e.g., average cost of therapeutic alternatives) influence physician practice behavior. The research design employed in this study made it impossible to measure the separate effects of information (report cards and profiles) alone versus counterdetailing education. The physician education-intervention can be multifaceted and when physician profile reports are coupled with group education sessions, the resulting impact on prescribing behavior can be synergistic. ${ }^{17,18}$ Such results are possible given that one of the more effective means of influencing physician prescribing behavior has been through face-to-face interactions with other health care professionals and pharmaceutical sales representatives. . $^{8,19}$

There may be other explanations for the findings in this study. For example, the sertraline market share was undoubtedly influenced beginning in 1998 when physicians were encouraged to prescribe sertraline and have patients split the 100mg tablets. Part of the health plan physician education-intervention during this time included the observation that health plan members could potentially save a monthly copayment by splitting sertraline tablets and the health plan's net sertraline costs were nearly halved for the patients who could split tablets. Pharmacy claims data did not allow for accurate evaluation of how patients were instructed to take their medications, and so, we are unable to quantify the effects of pill-splitting of sertraline.

This study did not differentiate between clinics that received the reports with academic detailing from those that received the reports through the mail. The clinics that did not receive academic detailing were very small in size (6\% of all family practice and internal medicine physicians with approximately 6,000 members or about $4 \%$ of total health plan membership). It is unlikely that the results of this study would change significantly as a result of not including these few practitioners and patients in the intervention program. As noted previously, some physicians practiced at sites with academic detailing and at sites without academic detailing, making difficult any evaluation of individual clinic performance.

The effectiveness of physician profiles can be hampered by the physician lack of confidence in the validity or reliability of the information contained in these reports. However, in this health plan, any questions raised by physicians regarding the accuracy of prescriptions documented on the physician profile reports were further investigated and changes to the reports were made accordingly. Departmental data may have also been affected by changes in the number of physicians practicing at the regional clinic.

Other limitations of this research also exist. Some patients had more than one health plan identification number. This identifier was used in calculating the number of SSRI utilizing members (patients). It was not possible to merge prescription claims for the same patient across different identifiers. Also, this study did not differentiate between the 13 clinics that received the reports through academic detailing from the six clinics that received the reports through the mail. Lastly, it was not possible to have a control group of physicians for comparison since all physicians employed by the health plan received quarterly physician profiles. Such opportunities for comparison with physicians that did not receive reports would allow for more accurate evaluation of the efficacy of the profiles in decreasing SSRI expenditures.

National data may reflect MCO mechanisms to shift market share through copay incentives or formulary status or therapeutic interchange programs. Although an exact comparison between this health plan and national data may be imperfect, the decline in the market share for fluoxetine was more rapid than the national trend after the physician profiles and counterdetailing intervention were implemented in this Health plan.

As physician confidence in these reports improves, profiles can be used to target other therapeutic areas and drug classes including hypertension, diabetes, congestive heart failure, and gastrointestinal disorders. Additionally, clinical information (patient-specific or general) can be incorporated into physician profiles, such as hemoglobin Alc or microalbuminuria in diabetic patients. Finally, physician profiles can include potential cost-savings for using formulary or generic alternatives or be used for physician performance evaluations.

\section{Conclusions}

Per member per year utilization and expenditures for fluoxetine decreased despite the increased overall number of SSRI prescriptions and SSRI utilizing members (patients) in CY 1999 and CY 2000. Due to this change in fluoxetine utilization, and the coincident market share shift to less costly SSRIs, the average SSRI cost per day of therapy declined by $7.4 \%$, in constant dollars, from $\$ 2.43$ per day of SSRI therapy in CY 1998 to $\$ 2.25$ per day of therapy in CY 1999 and by an additional 4.0\% to $\$ 2.16$ per day of SSRI therapy in CY 2000. The health plan market share of fluoxetine declined by $50 \%$, from $46.2 \%$ of prescriptions in 1997 to $23.0 \%$ of prescriptions in 2000, compared to national Rx share data which showed $31 \%$ erosion in relative fluoxetine Rx market share during this time period, from $44.6 \%$ of Rxs in 1997 to $30.6 \%$ of Rxs in 2000. Market share, as meas- 
Effect of Physician Profiles and Academic Detailing on Cost and Utilization of Selective Serotonin Reuptake Inhibitors

ured by SSRI days of therapy and SSRI patients, paralleled the $50 \%$ decline in relative fluoxetine utilization in this health plan. The combined intervention program of physician education employing profile-report cards and counter-detailing emphasizing the relative cost of SSRI therapeutic alternatives was associated with incremental two-year savings of $\$ 206,644$ or an average of $\$ 0.61$ per health plan member per year.

\section{Disclosure}

Study concept and design were contributed primarily by Godley, Doan, Yokoyama, Pham, Jackimiec, Woodward, and Clanton. The manuscript draft was prepared by Yokoyama, Doan, and Godley. Analysis and interpretation of data was conducted by Yokoyama, Doan, Godley and Jackimiec. They also were primarily responsible for critical revisions along with Pham. At the time of the study, Yokoyama and Doan were research fellows for Novartis Pharmaceuticals Corporation. The study was conducted independent of Novartis Pharmaceuticals Corporation.

\section{References}

1. Balas EA, Boren SA, Brown GD, et al. Effect of physician profiling on utilization. Meta-analysis of randomized clinical trials. J Gen Intern Med. 1996; 11: $584-590$

2. Gehlbach SH, Wilkinson WE, Hammond WE, et al. Improving drug prescribing in a primary care practice. Med Care 1984; 22(3): 193-201.

3. Hamley JT, Brown SV, Crooks J, et al. Prescribing in general practice and the provision of drug information. Journal of the Royal College of General Practitioners 1981; 31: 654-60.

4. Berwick DM, Coltin KL. Feedback reduces test use in a health maintenance organization. JAMA. 1986; 255: 1450-54.

5. Schectman JM, Kanwal NK, Schroth WS, et al. The effect of an education and feedback intervention on group-model and network-model health maintenance organization physician prescribing behavior. Med Care. 1995; 33: 139-44.
6. Hershey CO, Goldberg HI, Cohen DI. The effect of computerized feedback coupled with a newsletter upon outpatient prescribing charges. Med Care. 1988; 26: 88-94

7. Brufsky JW, Ross-Degnan D, Calabrese D, et al. Shifting physician prescribing to a preferred histamine-2-receptor antagonist: effects of a multifactorial intervention in a mixed-model health maintenance organization. Med Care. 1998; 36: 321-32

8. Steele MA, Bess DT, Franse VL, et al. Cost-effectiveness of two interventions for reducing outpatient prescribing costs. DICP Ann Pharmacother. 1989; 23: 497-500.

9. Wones RG. Failure of low-cost audits with feedback to reduce laboratory test utilization. Med Care. 1987; 25: 78-82.

10. Emmons DW, Wozniak GD. Profiles and feedback: Who measures physician performance? In: Gonzales ML, ed. Socioeconomic Characteristics in Medical Practice. 1994. Chicago, Ill.: American Medical Association; 1994.

11. Emigh RC, ed. Novartis Pharmacy Benefit Report: Facts \& Figures. 1999 ed. East Hanover (NJ): Novartis; 1999.

12. Emigh RC, ed. Novartis Pharmacy Benefit Report: Facts \& Figures. 2000 ed. East Hanover (NJ): Novartis; 2000

13. Emigh RC, ed. Novartis Pharmacy Benefit Report: Facts \& Figures. 2001 ed. East Hanover (NJ): Novartis; 2001.

14. Bureau of Labor Statistics. (2001). Consumer Price Indexes Home Page [WWW page]. URL: http://stats.bls.gov/cpihome.htm.

15. Express Scripts 1999 Drug Trend Report. Maryland Heights (MO): Express Scripts; 2000

16. Express Scripts 2000 Drug Trend Report. Maryland Heights (MO): Express Scripts; 2001.

17. Davis DA, Thomson MA, Oxman AD, Haynes RB. Changing physician performance: a systematic review of the effect of continuing medical education strategies. JAMA 1995; 274(9): 700-705.

18. Fox RD, Mazmanian PE, Putnam RW. Changing and learning in the lives of physicians. New York: Praeger, 1989.

19. Soumerai SB, McLaughlin TJ, Avorn J. Improving drug prescribing in primary care: a critical analysis of the experimental literature. Milbank Q. 1989; 67: 268-317. 